\title{
Radio Resource Management in Cloud Computing Based Cognitive Radio
}

\author{
Khatimiti Saad Waqqas (Mescoe Pune)
}

\begin{abstract}
Cognitive Radio Network has emerged as a solution to the growing spectrum scarcity and inefficiency problems. However, Cognitive Radio Networks face performance and security bottlenecks due to lack of memory and vast computational capabilities. This problem could be solved if we make use of Cloud as a central entity for storing spectrum availability information and processing of the spectrum availability data and correctly map the location of the unlicensed user to that of the available spectrum bands. We will be considering only those spectrum bands for communication where the primary users are absent. If the licensed user is detected, we shall empty that band and move to another idle spectrum band that matches our requirements. Admittance will be based on FCFS basis and at the same time the Quality of Service requirements (in terms of data rate) of the unlicensed users will satisfied.
\end{abstract}

\section{Keywords:}

\section{Introduction}

In the PAST decade, Software Defined Radio (SDR) and Cognitive Radio (CR) technology has revolutionized our view of opportunities in wireless communications to a great extent. The key motivation behind this technology is to increase spectral utilization and to optimize the use of radio resources. As SDR and CR are clearly emerging as a strong technological opportunity, research and development is being promoted rapidly throughout the wireless industry and in the academic research arena. Correspondingly, the standardization, regulation and certification activities are also being initiated in many parts of the world including IEEE 802.22, Wireless Innovation Forum and ETSI. However, the security issues on SDR and CR is still under research especially for commercially viable prototypes and future products and its implications on standardization.SDR technology implements radio functionalities like modulation/demodulation, signal generation, signal processing and signal coding in software instead of hardware as in conventional radio systems. The software implementation provides a higher degree of flexibility and reconfigurability and many benefits including the capability to change the channel assignments, to change the provided communication services or modify the transmission parameters or communication protocols. SDR is also considered a technology enabler for $\mathrm{CR}$, which are intelligent" radios, which can learn from the environment and adapt their transmission/reception frequencies and parameters to improve spectrum utilization and communication efficiency. SDR and CR technologies are fundamental blocks to provide a more flexible approach to spectrum management in comparison to the conventional approach where radio frequency spectrum bands are statically allocated by spectrum regulators.

The fixed spectrum assignment policies rule today's wireless network. This means that the spectrum is regulated by governmental agencies and is assigned to license holders or services on a long term basis for large geographical regions. This leads to sporadic usage of the spectrum and concentration of signal strength in certain portions while significant amount of spectrum is left unused. According to Federal Communications Commission (FCC), there exist temporal and geographical variations in the utilization of the assigned spectrum. The utilization ranges from $15 \%$ to $85 \%$. The problems with the spectrum assignment policy started recently with the growth of usage of wireless networks and mobile services. These problems have resulted in need for Dynamic Spectrum Access to exploit the spectrum opportunistically. For Dynamic Spectrum Access we can make use of Cognitive Radio Networks. However, the limited memory and computational capacity of Cognitive Radio devices result in decreased performance making their realization on global basis impractical.

It is well known that security is an important element in wireless communications. While SDR and CR based systems should guarantee the same level of security of

Conventional wireless communication systems, they may also present new vulnerabilities or security threats. As a general rule, communication systems based on SDR and CR technology must validate communication security requirements like Data Confidentiality and Privacy, Availability, Registration, Authentication and Authorization. This is a consequence of the general conformance to standards and regulations already defined for the wireless communication systems, with which SDR and CR devices must interoperate. For example, if SDR and CR devices are used in the public safety domain, they should satisfy the government approved security requirements defined by the TETRA or APCO 25 standards. SDR and CR concepts may provide new powerful capabilities but they may also be vulnerable to new types of security attacks, beyond the ones already defined for conventional networks.

\subsection{Cloud Computing}

The fixed spectrum assignment policies rule today's wireless network. This means that the spectrum is regulated by governmental agencies and is assigned to license holders or services on a long term basis for large geographical regions. This leads to sporadic usage of the spectrum and concentration of signal strength in certain portions while significant amount of spectrum is left unused. According to Federal Communications Commission (FCC) there exist temporal and geographical variations in the utilization of the assigned spectrum. The utilization ranges from $15 \%$ to $85 \%$. The problems with the spectrum assignment policy started 


\section{International Journal of Science and Research (IJSR) \\ ISSN (Online): 2319-7064}

Index Copernicus Value (2013): 6.14 | Impact Factor (2014): 5.611

recently with the growth of usage of wireless networks and mobile services. These problems have resulted in need for Dynamic Spectrum Access to exploit the spectrum opportunistically. For Dynamic Spectrum Access we can make use of Cognitive Radio Networks. However, the limited memory and computational capacity of Cognitive Radio devices result in decreased performance making their realization on global basis impractical. To solve this problem, we propose the use of Cloud Services. The details of what is exactly cognitive Radio Network, what is Cloud Computing and how we can use it to implement the Dynamic Spectrum Access are given below.

\subsection{Objective}

1) Determine which portions of the spectrum are available and detect the presence of licensed users when a user operates in a licensed band (spectrum sensing).

2) Select the best available channel (spectrum management) for communication

3) Coordinate access to this channel with other users (spectrum sharing).

4) Vacate the channel when a licensed user is detected (spectrum mobility).

\section{Cognizant Radio}

The concept of SDR has evolved over several years. Over this period a number of references have provided their definition of SDR, which has given rise to various interpretations of what SDR actually is and what is not. A key reason is that SDR is an all embracing term, which may be applied to a wide range of radio platforms and concepts. SDR is defined as radio in which the radio frequency (RF) operating parameters including, but not limited to, frequency range, modulation type, or output power can be set or altered by software, and/or the technique by which this is achieved". An alternative definition is provided by the SDR Forum, now named Wireless Innovation Forum, which has developed a definition of SDR in cooperation with IEEE working group P1900.1:

- Raib in which some or all of the physical layer functions are software defined".

The Forum explains: -AnSDR defines a collection of hardware and software technologies where some or all of the radio's operating functions (also referred to as physical layer processing) are implemented through modifiable software or firmware operating on programmable processing technologies. These devices include field programmable gate array (FPGA), digital signal processors (DSP), general purpose processor (GPP), programmable system on chip (SoC) or other application specific programmable processors. The use of these technologies allows new wireless features and capabilities to be added to existing radio systems without requiring new hardware". Figure 1 provides a potential architecture of a SDR and its main elements starting from the Real Time Operating System (RTOS). The Software Framework provides basic functions and libraries to support the waveforms and their portability including the middleware. An example of software framework is the combination of Software Communications
Architecture (SCA) and CORBA middleware described in The waveform represents the software implementation ofa communication service (e.g. UMTS). Finally, applications can be defined to support a specific operational or businesscontext.CR is defined as radio, which has the following capabilities: to obtain the knowledge of radio operational environment and established policies and to monitor usage patterns and users' needs; to dynamically and autonomously adjust its operational parameters and protocols". The design and deployment of CR and DSA have been investigated in a number of papers and research studies starting from the paper of Joseph Mitola

It is usually recognized that CRs should provide the following functions:

1) Determine which portions of the spectrum are available and detect the presence of licensed users when a user operates in a licensed band

2) Select the best available channel for communication

3) Coordinate access to this channel with other users

4) Vacate the channel when a licensed user is detected

These functions are dependent on each other as described in Figure 2. The figure describes also the relationships among the functions. For example: spectrum mobility can alert the spectrum sensing function on detected changes in the spectrum environment. Acting on the alert, the spectrum sensing function can collect again the knowledge of the spectrum environment and provide it to the spectrum management function to re-plan the allocation of spectrum bands. The disruption of spectrum sensing has an impact on the other functions because they will not have the needed information to perform effectively. Spectrum management requires the knowledge of the spectrum environment acquired by spectrum sensing to select the best available channel. Spectrum sharing needs the information on the bands selected by spectrum management function in each node. Spectrum mobility needs information on spectrum environment changes from spectrum sensing and the current allocation of spectrum bands from spectrum management and spectrum sharing.CR networks are composed by many nodes, with different capabilities, which interact on the basis of defined protocols and policies. To implement and deploy DSA, CR nodes do not operate in isolation but they are part of wider CR networks, which must provide a higher set of capabilities, which include context awareness and resource management. Context awareness means that a node or a network should be aware of the operational context, existing policies and regulations, network and spectrum awareness and user requirements in terms of requested traffic capacity, quality of service (QoS), resilience and security. Resource management means that $\mathrm{CR}$ nodes must cooperate to allocate the available network or spectrum resources, not only internally but also externally to other conventional networks. Each of these capabilities can be disrupted by intentional or unintentional threats. For example: a malicious attacker can implement a security attack against the resource management capability to allocate network or spectrum resources to itself or simply to provoke a Denial of Service (DoS) activity. 


\section{International Journal of Science and Research (IJSR) \\ ISSN (Online): 2319-7064}

Index Copernicus Value (2013): 6.14 | Impact Factor (2014): 5.611

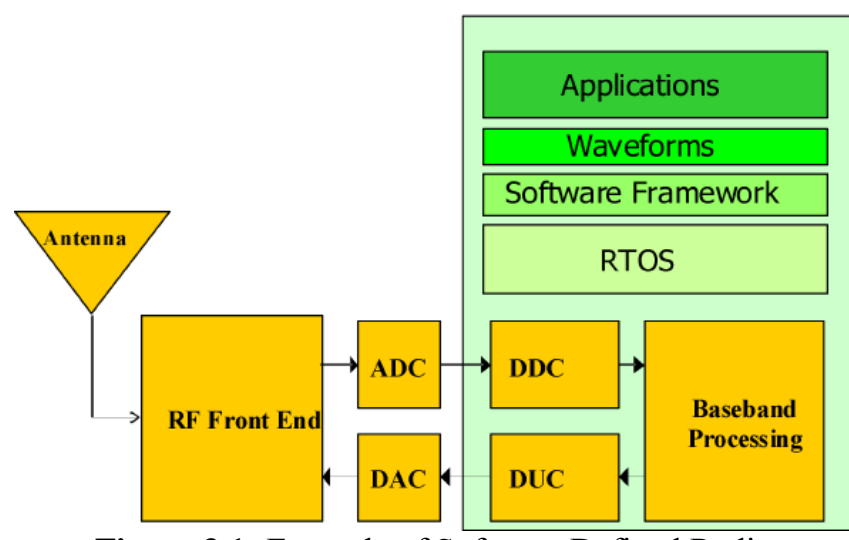

Figure 3.1: Example of Software Defined Radio architecture; ADC (analog todigital conversion), DAC

(digital to analog conversion), DDC (digital

downconversion), DUC (digital up conversion).

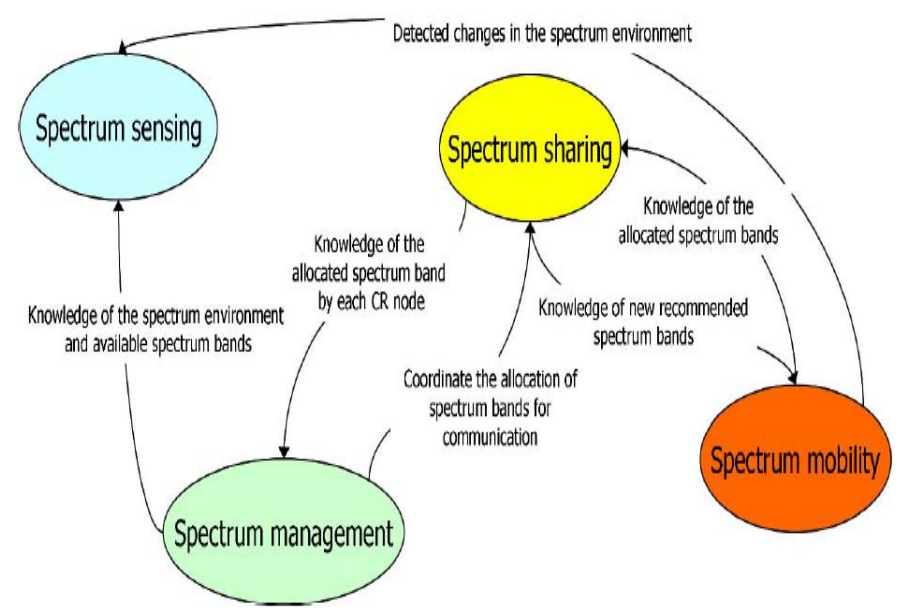

Figure 3.2: Dependencies among cognitive radio functions

The two most common approaches are collaborative and uncooperative:

1) In the collaborative approach, the cognitive functions are based on the coordination of the CR nodes, which exchange information to optimize the spectrum utilization and to improve the efficiency of the network.

2) In the uncooperative approach, each CR node implements the cognitive functions on its own.

The collaborative approach is usually considered more efficient, faster to converge to shared spectrum resources allocation and more reliable than the uncooperative approach but it requires common channels to exchange information. The common channel is often called Cognitive Control Channel (CCC) and it is responsible for distributing the cognitive messages in the $\mathrm{CR}$ network. In turn, the collaborative approach can be centralized or distributed. In a centralized solution, a central node, e.g. a base station (BS), controls the allocation of the spectrum resources or collects the spectrum sensing information. In the distributed solution, the CR nodes must agree on a common spectrum allocation through decision algorithms or voting systems. A centralized solution may be more efficient but the central node can represent a single point of failure. A comparison between the centralized and distributed approaches for spectrum management is presented in. Furthermore, a centralized solution may imply the presence of an existing infrastructure or pre-existing contracts to identify the centralized node.
Each of the proposed CR architectures has different levels of vulnerability against specific security threats.

\section{Why Cognitive radio}

With wire-less and radio communications becoming far more widely used, and the current levels of growth looking to increase, ideas such as cognitive radio will become more important. Some areas of the spectrum are very heavily used while others are relatively free.

Additionally the ability to change modes, frequencies and power levels will not only make communication possible for the cognitive radio system itself, but should also reduce the overall levels of interference to other users.

This is because the most spectrum or interference efficient modes can be chosen by the cognitive radio system.

In view of the possibility of $\mathrm{CR}$ radio communications systems utilising the spectrum more efficiently some regulatory bodies such as the FCC in the USA and Ofcom in the UK are looking favourably at the idea of cognitive radio. When the idea becomes a reality it would enable greater efficient use of the radio spectrum, which is not an infinite resource as it once was considered. Accordingly the way may be opened from this viewpoint to assist the development of cognitive radio communications technology.

\section{Cognitive Radio Parameters and Objectives}

In CR Network systems, the environmental parameters are defined as inputs to the CR system whereas the transmission parameters will be the system outputs. The relationships between the environmental and transmission parameters are formed by mathematical equations that are further defined as objective functions. As the cognitive radio systems senses the environment and reconfigure its transmission parameters optimally to satisfy the objectives and efficiently utilize the available spectrum band choosing of the best possible set of parameters is most important as it largely affect the accuracy and efficiency of CR. This can be done by determining the appropriate parameters and objectives for the system.

\section{Transmission Parameters}

Transmission parameters act as the decision variables for the CR system, so it must be well-defined before developing fitness functions for various objectives. Table 1 shows the list of transmission parameters used in our work.

TABLE 1 Transmission parameters

\begin{tabular}{|l|l|}
\hline PARAMETER NAME & DESCRIPTION \\
\hline Transmit power & Raw Power Transmission \\
\hline Modulation Type & Type of the modulation scheme used \\
\hline Modulation level & $\begin{array}{l}\text { No. of symbols used for given } \\
\text { modulation scheme }\end{array}$ \\
\hline Symbol rate & No. of symbols per second \\
\hline Packet size & Size of the packet \\
\hline
\end{tabular}

\section{Environmental Parameters}

Environmental parameters are the sensed information that provides knowledge about the surrounding environment's characteristic to the CR system. This helps in decision

\section{Volume 5 Issue 1, January 2016}




\section{International Journal of Science and Research (IJSR) \\ ISSN (Online): 2319-7064}

Index Copernicus Value (2013): 6.14 | Impact Factor (2014): 5.611

making process. The environmental parameters used here are: Data rate, Power consumption, Spectral efficiency, occupied band, Bit error and Packet error.

\section{Cognitive Radio Objectives}

Certain objectives needs to be fulfilled by the CR system are stated here. In this work, we will define the following objective functions in order to guide the system to an optimal state. Table 2 depicts the objectives used in this work.

The search space is created by combining the transmission and environmental parameters along with the defined objective functions. The range of the input transmission parameter is given in Table 3. The combination of these parameters with the objective function would lead to a large number of solutions which forms the search space.

TABLE 2 CR User's Objectives

\begin{tabular}{|l|l|}
\hline OBJECTIVES & DESCRIPTION \\
\hline $\begin{array}{l}\text { Optimum global } \\
\text { solution }\end{array}$ & Provides an optimal solution \\
\hline $\begin{array}{l}\text { Minimize } \\
\text { power } \\
\text { consumption }\end{array}$ & $\begin{array}{l}\text { Decrease the amount of power } \\
\text { consumed by the system }\end{array}$ \\
\hline $\begin{array}{l}\text { Maximize } \\
\text { throughput }\end{array}$ & $\begin{array}{l}\text { Increase the overall data throughput } \\
\text { transmitted by the radio }\end{array}$ \\
\hline Minimize BER & $\begin{array}{l}\text { Improve the overall BER of the } \\
\text { transmission environment }\end{array}$ \\
\hline
\end{tabular}

TABLE 3 Parameters range

\begin{tabular}{|l|l|}
\hline PARAMETERS & RANGE \\
\hline Transmit power & 1 to $30 \mathrm{~dB}$ \\
\hline Modulation Type & $\begin{array}{l}\text { BPSK, BFSK, MPSK, MQAM, } \\
\text { QSK, GMSK }\end{array}$ \\
\hline Modulation level & $2,4,8,16,32,64$ \\
\hline Symbol rate & $1 \mathrm{e} 4: 1 \mathrm{e} 3: 1.28 \mathrm{e} 5$ \\
\hline Packet size & $256: 16: 2048$ \\
\hline
\end{tabular}

Fitness Evolution for Cognitive Radio System

In this paper, four objective functions have been formulated to accomplish the four distinct objectives- optimal Global Minimum, Minimum power, Maximum Throughput and Minimum BER. Their respective objective function is given below:

The fitness function of minimizing power consumption is given as:

f min - power $=\mathrm{P} * \mathrm{Rs} * \mathrm{~K}$

Where, $\mathrm{P}$ is the transmitting power, Rs is the symbol rate, $\mathrm{K}$ is modulation index $(\mathrm{K}=2$ for MQAM and BPSK and $\mathrm{K}=1$ for remaining all).

The fitness function for maximizing data rate is given as:

f Max-Throughput $=$ Rs $* \log 2(\mathrm{M})$

Where Rs is the symbol rate, $\mathrm{M}$ is modulation index.

The fitness function for minimizing BER is given as:

$\mathrm{f}$ Min $-\mathrm{BER}=$ qfunc $\left(\operatorname{sqrt}\left(2.0^{*} \mathrm{ebno}\right)\right)$ forBPSK \& QPSK $\mathrm{f}$ Min- BER $=$ qfunc $($ sqrt (ebno) $)$ for BFSK

f Min- BER=qfunc $((2 * \log 2(\mathrm{M}) *$ ebno $) * \sin (\mathrm{pi} / \mathrm{M}))$ for MPSK

f Min- BER $=$ cef $*$ erfc $($ sqrt $(s))$ for MQAM

f Min- BER $=$ qfunc $($ sqrt $(2.0 *$ alpha*ebno) $)$ for GMSK

Where,

ebno $=\mathrm{CbN}+10 * \log 10(\mathrm{Bw} / \mathrm{Rb})$

The fitness function for minimizing Packet erroris given as: f Min- PER=1.0-(1.0-BER $)^{\wedge} \mathrm{Ps}$

Where, Psis Packet size.

The fitness function foroccupied bandwidthis given as:

$\mathrm{f} \mathrm{Bt}=(1+\mathrm{r})^{*} \mathrm{Rb}$ forBPSK\& FPSK

$\mathrm{f} \mathrm{Bt}=((1+\mathrm{r})) / \log 2(\mathrm{M})) * \mathrm{Rb}$ for MPSK\&MQAM

$\mathrm{fBt}=((1+\mathrm{r})) 2) * \mathrm{Rb}$ for QPSK

$\mathrm{f} \mathrm{Bt}=\mathrm{r} * \mathrm{Rb}$ for $\mathrm{GMSK}$

Where, $r$ isroll off factor and Rbis bitrate.

The fitness function formaximizing Spectral efficiencyis given

as:

$\mathrm{f}$ Max - spectraleff $=\mathrm{Rb} / \mathrm{BW}$

$\mathrm{BW}=1 \mathrm{e} 6$

Where, Rbis bit rate,

BWis band width.

The weighted sum approach has been used in this SA based CR system. The weighted sum approach allows us to combine the single objective functions into one aggregate multiple objective functions. fitness value $=\mathrm{ws}(1) * \mathrm{fMax}-$ Throughput+ws(2)*fMin-Power +ws(3)*fMax- spectraleff $+\mathrm{ws}(4) * \mathrm{fBt}+\mathrm{ws}(5) * \mathrm{fMin}-\mathrm{BER}+\mathrm{ws}(6) * \mathrm{fMin}-\mathrm{PER}$

Table 4 Weighting factors

\begin{tabular}{|l|l|l|}
\hline Scenarios & Weight Factors [w1 w2 w3 w4 w5 w6 $]$ \\
\hline $\begin{array}{l}\text { Global } \\
\text { minimum }\end{array}$ & {$\left[\begin{array}{llllll}0.166 & 0.166 & 0.166 & 0.166 & 0.166 & 0.166\end{array}\right]$} \\
\hline $\begin{array}{l}\text { Minimizing } \\
\text { Power } \\
\text { Consumption }\end{array}$ & {$\left[\begin{array}{llllll}0.08 & 0.6 & 0.08 & 0.08 & 0.08 & 0.08\end{array}\right]$} \\
\hline $\begin{array}{l}\text { Minimizing } \\
\text { BER }\end{array}$ & {$\left[\begin{array}{lllllll}0.45 & 0.05 & 0.2 & 0.2 & 0.05 & 0.05\end{array}\right]$} \\
\hline $\begin{array}{l}\text { Maximizing } \\
\text { Throughput }\end{array}$ & {$\left[\begin{array}{lllllll}0.1 & 0.1 & 0.1 & 0.1 & 0.4 & 0.2\end{array}\right]$} \\
\hline
\end{tabular}

The weighting values, w1, w2, w3, w4, w5 and w6 determine the search direction for the optimizing algorithm. We have defined four weight vectors representing common scenarios a cognitive may be placed in. each weight vector listed in Table 4 emphasizes different objectives that lead the algorithm using this fitness function to develop a olution that relate to a specific objective.

\section{Cloud Computing}

According to the National Institute of Standards and Technology the definition of Cloud Computing is: -Cloud Computing is a model for enabling convenient, on-demand network access to a shared pool of configurable computing resources (for example, networks, servers, storage, applications and services) that can be rapidly provisioned

\section{Volume 5 Issue 1, January 2016}




\section{International Journal of Science and Research (IJSR) \\ ISSN (Online): 2319-7064 \\ Index Copernicus Value (2013): 6.14 | Impact Factor (2014): 5.611}

and released with minimal management effort or service provider interaction." Cloud Computing is an economic solution to tasks that require large amount of storage and fast and complex computational capacity.

Cloud will be our central entity that stores the channel data from sensors in database and processes the unlicensed user requests and responds accurately to these requests. In order to map the geo-location of unlicensed users and the idle spectrum bands, we will need a Searching algorithm that calculates the distance between the locations of idle bands and unlicensed users using the information fed by sensors in the database and decides which bands are suitable for allocation to that particular user. It simply requires calculation of distance between the center co-ordinates of idle spectrum band and co-ordinates of the geographical location of unlicensed user.

In this report we look at all three categories, however a very simplified way of differentiating these flavors of Cloud Computing is as follows:

SaaS applications are designed for end-users, delivered over the web.

PaaS is the set of tools and services designed to make coding and deploying. those applications quick and efficient. IaaS is the hardware and software that powers it all servers, storage, network, operating systems.

\section{Methodology and Implementation}

\section{Database}

From the scenario put forth so far, few things that are clear are: We are going to need sensors for every network to continuously send information about the idle spectrum bands and these sensors are expected to keep on updating this information so that the unlicensed users are presented with greater opportunities and are informed when to migrate to other bands in case the licensed users appear in their bands. All this also implies that we are going to need vast storage and lot many calculations to locate the idle bands. However it is necessary that only those idle spectrum bands be assigned to the unlicensed users which lie in the same geographic location. To make this possible we need to make entries of the idle bands in the form of their frequencies, geographic location and data rate. We have also considered how to determine whether how long a particular spectrum band can be; used so as not to interfere with the licensed users. This is done by our CAM which acts as our Time-ToLive factor. A smaller CAM value makes a channel less desirable. The maximum data rates we will consider according to $802.11 \mathrm{~b}$ and $802.11 \mathrm{~g}$ are $11 \mathrm{Mbps}$ operating in $2.4 \mathrm{GHz}$ and $54 \mathrm{Mbps}$ in $5 \mathrm{GHz}$. Cellular downlink peak rates will be $300 \mathrm{Mbps}$ and uplink peak rates will be $75 \mathrm{Mbps}$ for 3GPP LTE wireless communication. 3G wireless systems offer data rate less than $1 \mathrm{Mbps}$. Maximum upload data rate for satellite communication is $10 \mathrm{Mbps}$. $3 \mathrm{G}$ cellular system and satellite bands are excluded here since they cannot fulfill demanded data rates of unlicensed users. In the next section we have explained why we propose using Cloud services for storage and computation.

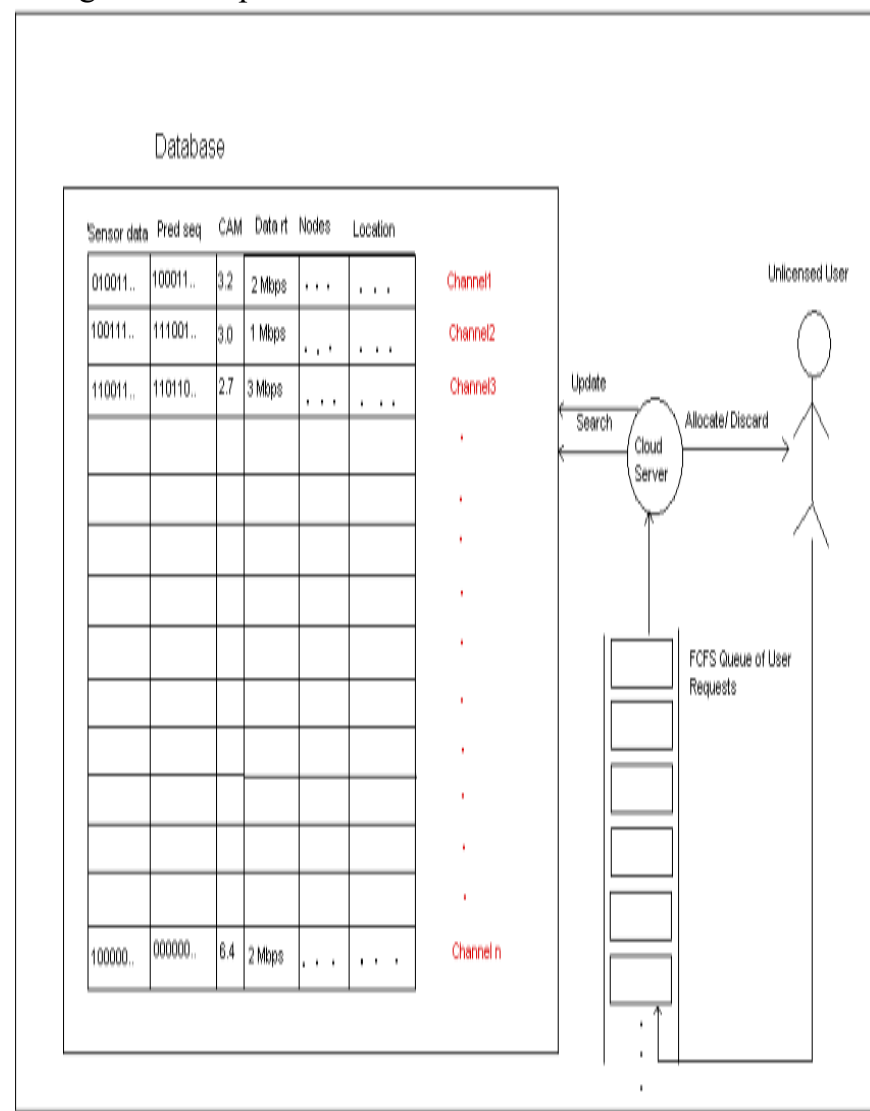

Figure 3.1: Database representation

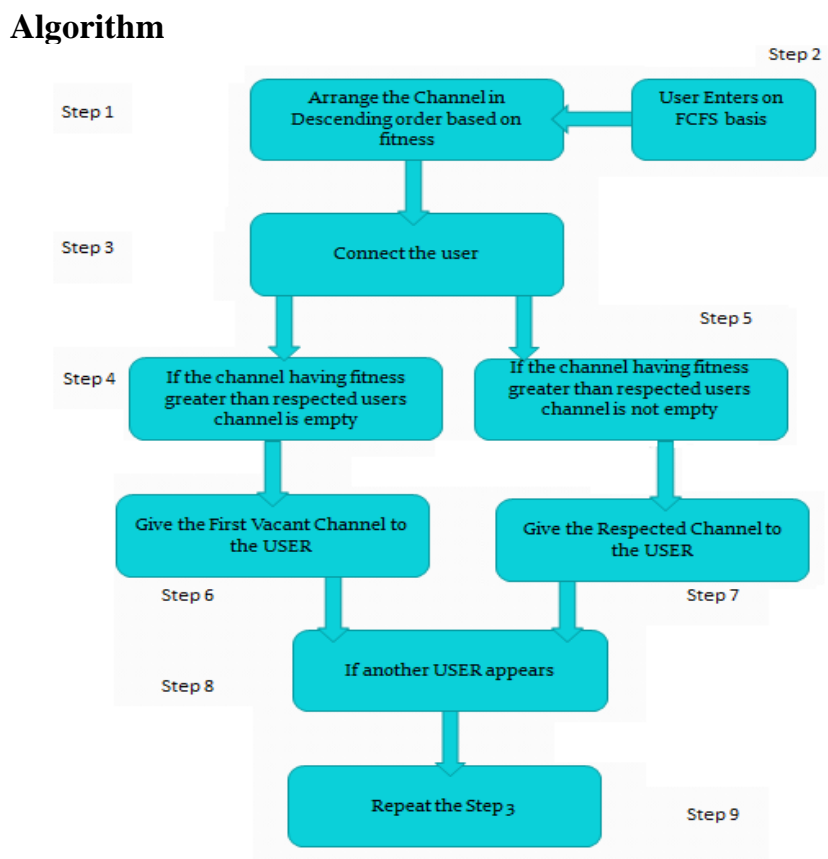

Results and Discussion 
International Journal of Science and Research (IJSR)

ISSN (Online): 2319-7064

Index Copernicus Value (2013): 6.14 | Impact Factor (2014): 5.611

RADIO RESOURCE MANAGEMENT IN CLOUD COMPUTING BASED COGNITIVE RADIO

\begin{tabular}{|c|c|c|c|c|c|c|c|c|c|c|c|}
\hline $\begin{array}{l}\text { User } \\
\text { Name }\end{array}$ & $\begin{array}{l}\text { Channel } \\
\text { Name }\end{array}$ & $\begin{array}{c}\text { Transmission Power } \\
\text { in (W) }\end{array}$ & $\begin{array}{l}\text { Modulation } \\
\text { Type }\end{array}$ & $\begin{array}{l}\text { Modulation } \\
\text { Level }\end{array}$ & $\begin{array}{l}\text { Symbol Rate } \\
\text { (kbps) }\end{array}$ & $\begin{array}{l}\text { Frequency } \\
(\mathrm{Hz})\end{array}$ & $\begin{array}{l}\text { Minimizing Power } \\
\text { Consumption }\end{array}$ & $\begin{array}{c}\text { Maximizing Data } \\
\text { Rate }\end{array}$ & $\begin{array}{l}\text { Final } \\
\text { Fitness }\end{array}$ & Connected & Connectedto \\
\hline Saad & $4 \mathrm{G}(\mathrm{LTE})$ & 50.12 & MQAM & 16 & 128 & 1800 & 1026.46 & 51.2 & 87.24 & $\checkmark$ & $4 \mathrm{G}(\mathrm{LTE})$ \\
\hline Hasan & $4 \mathrm{G}(\mathrm{LTE})$ & 50.12 & MQAM & 16 & 128 & 1800 & 1026.46 & 51.2 & 87.24 & $\square$ & \\
\hline Ashish & $4 \mathrm{G}(\mathrm{LTE})$ & 50.12 & MQAM & 16 & 128 & 1800 & 1026.46 & 51.2 & 87.24 & $\nabla$ & $4 \mathrm{G}(\mathrm{LTE})$ \\
\hline Mohsin & $3 \mathrm{G}$ & 1 & QPSK & 8 & 127 & 2100 & 10.16 & 38.1 & 4.62 & $\nabla$ & $4 \mathrm{G}(\mathrm{LTE})$ \\
\hline Kuljeet & $3 \mathrm{G}$ & 1 & QPSK & 8 & 127 & 2100 & 10.16 & 38.1 & 4.62 & $\nabla$ & $3 \mathrm{G}$ \\
\hline Nabeel & $3 \mathrm{G}$ & 1 & QPSK & 8 & 127 & 2100 & 10.16 & 38.1 & 4.62 & $\nabla$ & $3 \mathrm{G}$ \\
\hline Amin & $2.5 \mathrm{G}$ & 0.63 & GMSK & 8 & 126 & 1800 & 6.35 & 37.8 & 4.29 & $\square$ & \\
\hline Gufran & $2.5 \mathrm{G}$ & 0.63 & GMSK & 8 & 126 & 1800 & 6.35 & 37.8 & 4.29 & $\nabla$ & $3 \mathrm{G}$ \\
\hline Umair & $2.5 \mathrm{G}$ & 0.63 & GMSK & 8 & 126 & 1800 & 6.35 & 37.8 & 4.29 & 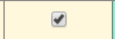 & $2.5 \mathrm{G}$ \\
\hline Anis & $2 \mathrm{G}$ & 0.4 & GMSK & 8 & 126 & 900 & 4.03 & 37.8 & 4.1 & $\square$ & $2.5 \mathrm{G}$ \\
\hline Afsar & $2 \mathrm{G}$ & 0.4 & GMSK & 8 & 126 & 900 & 4.03 & 37.8 & 4.1 & $\nabla$ & $2.5 \mathrm{G}$ \\
\hline Suman & $2 \mathrm{G}$ & 0.4 & GMSK & 8 & 126 & 900 & 4.03 & 37.8 & 4.1 & $\nabla$ & $2 \mathrm{G}$ \\
\hline
\end{tabular}

Guided by Nabegha Masood

By Saad Khatimiti

MESCOE PUNE

The above Application is developed in angularjs. Here only the simulation is done how the cognitive radio works Cognitive radio resources are also mentioned. Status of the channel is shown. Channels are arrange in descending order . The column -onnected" shows that the user is connected. The column -eonnected to" shows the actual cognizant. It also show the channel is connected to which channel.

There are 4channel such and 12 user accordingly. Admin can add channel and user as many they want. The channel details are shown in term of parameter. The parameter are characterized in environmental and transmission parameter.

The Transmission parameter are

1) Transmission power

2) Modulation Type

3) Modulation Level

4) Symbol Rate

5) Frequency

The Environmental parameter are

1) Minimizing Power Consumption

2) Maximizing Data Rate

Considering the above parameter and calculating final fitness factor. Managing channel accordingly

\section{Advantages and Application}

\section{Cognizant Radio}

Receive broadcast radio

Ettus Research, the folks behind what has come to be regarded as the grand addy of affordable wideband SDR hardware platforms: This is based on using GNU Radio and its excellent graphical tool, GNU Radio Companion (GRC).Of course, you don't need hardware anywhere near as capable as a USRP for this, and the low cost RTLSDR receiver hardware

Amateur radio

As you might expect radio hams are doing a lot of work with SDR and there are plenty of options available. Ranging from the popular, low cost and simple to understand Soft Rock SDR hardware, up to the modular and incredibly flexible $\underline{\text { High Performance Software-Defined Radio (HPSDR) }}$ project .In addition to using hardware that has been designed with amateur radio use in mind, it's also possible to use something such as a USRP or RTLSDR receiver, depending on whether you need a transceiver or receive-only, and the required frequency coverage and dynamic range etc. When it comes to software there are applications which are based on GNU Radio, such as the popular Gqrx receiver, along with many others that are based on the much simpler DttSP project.

Track ships via AIS transmissions

Automatic Identification System (AIS) is an automatic tracking system employed by ships to identify and locate vessels, which is used to supplement marine radar.

There are a number of options available for receiving and decoding AIS data, and one which uses RTLSDR hardware with a GNU Radio-based receiver plus gnuais is described in a blog post by Alexandru Csete, who also happens to be the author of the aforementioned Gqrx software. Using this AIS messages can be logged, plotted, and fed to the Google Maps-basedaprs.fi service

Track aircraft via Mode S transmissions

Mode $\mathrm{S}$ is similar in purpose to AIS, albeit for aircraft. Once again the humble RTLSDR hardware can be used for receiving transmissions, The air-modes software can be used for this purpose and with aircraft positions plotted in Google Earth.

\section{Set up a DRM transmitter}

That's not DRM as in Digital Rights Management, but rather instead Digital Radio Mondiale - the set of digital radio technologies designed for use with shortwave AM broadcasting. A DRM implementation was created by a student as part GNU Radio's participation in Google Summer of Code 2012. The gr-drm software is fully integrated with GNU Radio Companion and together with a USRP can be used to create a DRM/DRM+ transmitter. 


\section{International Journal of Science and Research (IJSR) \\ ISSN (Online): 2319-7064 \\ Index Copernicus Value (2013): 6.14 | Impact Factor (2014): 5.611}

Build a GSM network

When it comes to creating a GSM network using open source SDR there are two software options: Open BTS and Osmo BTS. The former has been around the longest and is used with a software switch such as Asterisk to essentially turn mobile handsets into SIP/VoIP endpoints. Osmo BTS provides layers 1-3 of a GSM base station and can be used with the transceiver component from Open BTS, to provide a fully open source implementation which may be used with Asterisk, or integrated with a traditional GSM network architecture using the Abis protocol.

Supported hardware includes USRP, development kit from Range Networks and UmTRX. The latter being a dualchannel open source hardware design that is designed for use in carrier networks.

\section{Experiment with LTE}

It's much earlier days as far as open source LTE (4G mobile) is concerned, but there are currently two partial implementations. gr-lte is a modular GNU Radio-based environment for an LTE downlink receiver, and open LTE provides GNU Octave code for test simulation along with GNU Radio applications. The latter includes downlink scanner and recorder applications which have hardware support for RTLSDR andHackRF.

\subsection{Cloud Computing}

Scalability - With increase in workload, the need for hardware and software increases which can be easily provided by Cloud computing without delay. Hence an organization can easily add or subtract cloud services and need not pay for anything more than what they use.

Easy Implementation - There is no need to purchase any hardware, software licenses or implementation services, an organization can easily hit the ground running by simply demanding cloud services in record time.

Skilled Practitioners - All sort of available technologies are provided and customized according to the requirements of clients by cloud providers without much delay no matter how popular that service is.

Frees up internal resources - As much of the work is assigned to third party providers, we are allowed to utilize our internal resources for other important tasks.

Quality of service - Cloud providers offer 24/7 services and immediate response to emergency situations to their clients.

\section{Conclusions}

In this we have presented opportunistic spectrum access by unlicensed user whose geo-location matches with the geolocation of the ideal licensed spectrum. Those unlicensed users who offer higher benefit are admitted. We have given the problem to maximize the profit and also satisfying the geo-location matching criteria. Malevolent unlicensed user can be tracked by geo-location matching. The lack of available spectrum and the simultaneous need to provision increasing number of applications, notably the bandwidth hungry variants, such as real-time video, is a driving force to explore spectrum sharing as an essential element of future wireless systems. As the discussion provided in this has indicated, a number of protection mechanisms are key to realizing an assured dynamic spectral environment to benefit the commercial, public-safety and military users.

\section{References}

[1] TajSturman,Abdur Rahim Biswas, RuedigerLeschhorn, Gy"oz"oG'odor, and Michael Street," Security Aspects in Software Defined Radio and Cognitive Radio Networks:A Survey and A Way Ahead", IEEE COMMUNICATIONS SURVEYS \& TUTORIALS, VOL. 14, NO. 2, SECOND QUARTER 2012

[2] Ashwini S Gulbhile, Mayur P Patil, Preeti P Pawar, Saloni V Mahajan, SunitaBarve,"Secure Radio Resource Management In Cloud Computing Based Cognitive Radio Network", INTERNATIONAL JOURNAL OF SCIENTIFIC \& TECHNOLOGY RESEARCH VOLUME 3, ISSUE 5, May 2014

[3] Understanding the Cloud Computing Stack: PaaS, SaaS, IaaS Diversity Limited, 2011 Non-commercial reuse with attribution permitted.

[4] GoutamGhosh, Prasun Das and SubhajitChatterjee'," Cognitive Radio And Dynamic Spectrum Access -A Study", International Journal of Next-Generation Networks (IJNGN) Vol.6, No.1, March 2014

[5] Asad A. Abidi', Evolution of the SoftwareDefinedRadio (SDR)Receiver", Electrical Engineering DepartmentUniversity of California, Los Angeles

[6] Lamiaa Khalid, AlaganAnpalagan', Emerging cognitive radio technology: Principles, challengesand opportunities"WINCORE Laboratory, Ryerson University, Toronto, Canada.

[7] Anita Garhwall and ParthaPratim Bhattacharya'," A SURVEY ON DYNAMIC SPECTRUM ACCESSTECHNIQUES FOR COGNITIVE RADIO International Journal of Next-Generation Networks (IJNGN) Vol.3, No.4, December 2011.

[8] Chunxiao Li, AnandRaghunathan, Niraj K Jha'," An Architecture for Secure Software Defined Radio", 9783-9810801-5-5/DATE09 (C) 2009 EDAA by microsoft',-Cofiguring and Deploying a Private Cloudwith System Center 2012" 